\title{
Renal perfusion phase curve analysis in DTPA renal scintigraphy - significance \& utility
}

\author{
V Sivasubramaniyan ${ }^{3}$, Osho Sai ${ }^{2}, \mathrm{~K}$ Venkataramaniah ${ }^{1}$ \\ ${ }^{1}$ Head of Department, Department of Nuclear Medicine, Sri Sathya Sai Institute of Higher Medical Sciences, Prashantinilayam, Anantapur \\ Dist., Andhra Pradesh, India, ${ }^{2}$ Nuclear Medicine Technologist, Department of Physics, Sri Sathya Sai Institute of Higher Learning, \\ Prashantinilayam, Anantapur Dist., Andhra Pradesh, India, ${ }^{3}$ Professor, Department of Physics, Sri Sathya Sai Institute of Higher Learning, \\ Prashantinilayam, Anantapur Dist., Andhra Pradesh, India,
}

\section{A B S T R A C T}

Aims and Objectives: The retrospective study of renal scans aims at analysing the renal perfusion phase curve in the DTPA scan. Four different types of perfusion phase curves for non-transplant kidney are defined and validated by comparing them with the Hilson perfusion index and Kirchner's K/A ratio. Materials and Methods: This study consists of 36 patients comprising of 26 males with mean age of $26.17 \pm 18.65$ and 10 females with the mean age of $33.20 \pm 11.69$. Renal scans were done using Siemens dual head gamma camera. 3-5mCi 99m Tc DTPA was used and injected intravenously, images were processed to obtain the renal curve using the icon software utilizing the Gate's formula. The perfusion curves, which form the part of renographic curves are taken for scrutiny, classified into different types and perfusion index was calculated. Results: The perfusion phase curves were scrutinised and categorised in 4 types. These curves have been validated by using Hilson index and Kirchner's K/A ratio. Hilson perfusion index and Kirchner's K/A ratio shows a good correlation with the uptake phase of conventional renal curve. We observed $47.22 \%$ of Type I, $22.22 \%$ of Type II, $16.67 \%$ of Type III and $13.89 \%$ of Type IV. Conclusion: This study highlights the utility of renal perfusion phase curve analysis in the non-transplant kidney. This can also serve as a better prognostic tool in the chronic kidney disease patient.

Key words: Renal scintigraphy, Renal perfusion phase curve, Hilson index, Kirchner's K/A ratio

Access this article online

Website:

http://nepjol.info/index.php/AJMS

DOI: 10.3126/ajms.v7i2.12136

E-ISSN: 2091-0576

P-ISSN: 2467-9100

\section{INTRODUCTION}

The analysis of the perfusion phase of the renal time activity curve (TAC) is the evaluation of choice in transplant kidneys. The Hilson perfusion index (PI) and Kirchner's K/A Ratio were the ones mostly utilised.

This study aims to characterise different types of renal perfusion phase curve as obtained from the GFR analysis in Siemen's icon software and its utility in the renal diseases.

The GFR was predicted from the serum creatinine (SCr) level at renography using GATES formula:

For men

$\operatorname{GFR}(\mathrm{ml} / \mathrm{min})=[(140-$ age $) \times$ weight $] /(\mathrm{SCr} \times 72)$
For women

$\operatorname{GFR}(\mathrm{ml} / \mathrm{min})=0.85 \times[(140-$ age $) \times$ weight $) /(\mathrm{SCr} \times 72)]$

Weight: body weight (kg)

SCr: serum creatinine level $(\mathrm{mg} / \mathrm{dl})^{1}$

The different types of perfusion curves are validated by using the following parameters:

Hilson Perfusion index \& Kirchner's K/A ratios, derived from the aortic artery and kidney region values.

\section{Perfusion index}

Amount of tracer reaching to the kidney through the blood is a tool for assessing renal function, various such tolls are available, most useful are Hilson perfusion index and Kirchner's K/A Ratio. 
Hilson perfusion index

Hilson perfusion index is the ratio of the area under the aortic curve divided by the renal perfusion curve.

$$
\text { Hilson Perfusion Index (HPI) }=\frac{\text { Area under the Aorta }}{\text { area under the Kidney }}
$$

Hilson et al. in $1978,{ }^{2}$ on the first application of their PI, studied 152 patients with 779 dynamic renal studies to evaluate this index. They selected only 276 studies based on the clinical status of the transplant which was defined retrospectively. The scintigrams were retrospectively divided into four groups: normal, ATN, rejection and renal artery stenosis according to the clinical diagnosis.

They mentioned that many studies (504 studies) could not be allocated to any group either because the clinical state was unclear or because it was in transition (for instance recovering from ATN or after treatment of rejection). The mean values of the PI for the different groups were 96 for normal grafts, 153 for the ATN group, 256 for the rejection group, and 255 for the patients with renal artery stenosis. They reported that the results of calculation of PI can differentiate clearly between normal grafts and those with impaired function, especially rejection cases. With good attention to the bolus injection the index has a standard error of $5 \%$, allowing early diagnosis of rejection and it can monitor the response to treatment in rejection episodes as it clearly declines in responding patients. $^{3}$

\section{Kirchner's K/A ratio}

This index is defined as the ratio of the slope of the maximum upstroke portions of the kidney and aortic curves. Kirchner et $\mathrm{a}^{\left[{ }^{[4]}\right.}$ on their first application of this index on $105\left[{ }^{99 \mathrm{~m}} \mathrm{Tc}\right] \mathrm{DTPA}$ scintigrams stated that this $\mathrm{K} / \mathrm{A}$ ratio has been of significant help in the differential diagnosis of early post-transplant anuria. Ratios were reported to be low in rejection, acute tubular necrosis (ATN) and obstruction and rose with recovery. This K/A ratio is highly dependent on the shape of the TACs and the characteristics of the bolus injection. In subsequent studies the authors themselves ${ }^{4,5}$ stated that $\mathrm{K} / \mathrm{A}$ ratio is proportional to renal blood flow only when the aortic TAC is of good quality.

The Hilson ratio has been used in this study to validate 4 different type of perfusion curves.

Kirchner's $\frac{\mathrm{K}}{\mathrm{A}}$ ratio $=\frac{\text { Area under the kidney }}{\text { Area under the aorta }}$

\section{SUBJECTS AND METHODS}

This was a retrospective study done on 36 patients, comprising of 26 males in the age range of 3 to 61 with mean age of $25.83 \pm 18.62$ and 10 females in the age range of 18 to 55 with the mean age of $33.20 \pm 11.69$. These patient were referred by urology department for differential renal functioning. The perfusion curves which forms part of the conventional renographic curves are taken for scrutiny, these curves were analysed and the area under the curve was calculated. The area under the different kidneys and aortic curves was calculated and stored, using the conventional Hilson index ${ }^{5}$ formula and Kirchner's K/A ratio $^{6-8}$, perfusion index was calculated for each kidney.

Depending upon their configuration following type of perfusion curves are proposed.

Type I perfusion curve has an initial straight line followed by the steep vertical rise followed by a plateau or horizontal line.

In the normal condition the aortic pressure will be more than renal artery pressure which will be more than segmental artery pressure. In this case we will get the ideal typical TYPE I curve ${ }^{9}$, as shown in the Figures 1 and 2.

TYPE I: AP > RAP > SAP

(AP-Aortic pressure; RAP-Renal artery pressure; SAP- Segmental artery pressure)

Type II perfusion curve has horizontal line followed by blunted slope followed by a genu and a plateau.

When the aortic pressure is greater than renal artery pressure and the segmental artery pressure is greater than or equal to renal artery pressure than we will get a typical TYPE II curve9, as shown in the Figures 2 and 3.

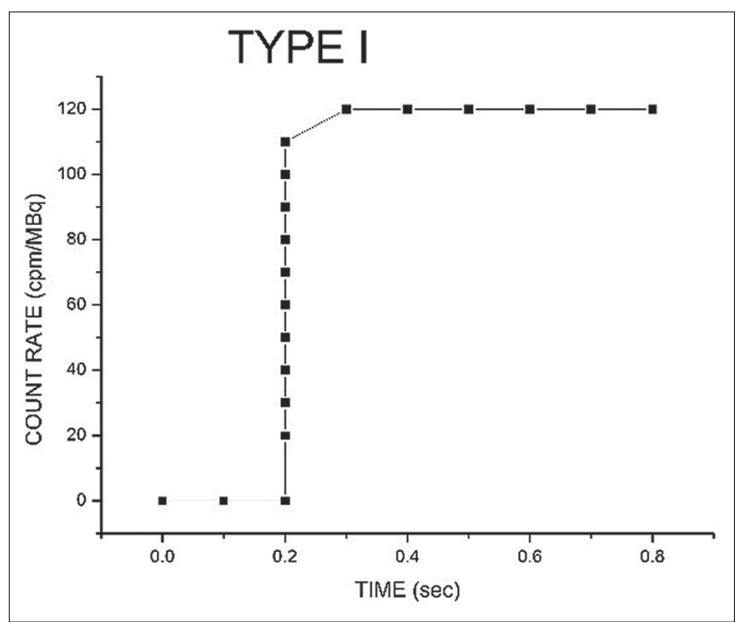

Figure 1: Ideal Type I perfusion phase curve 


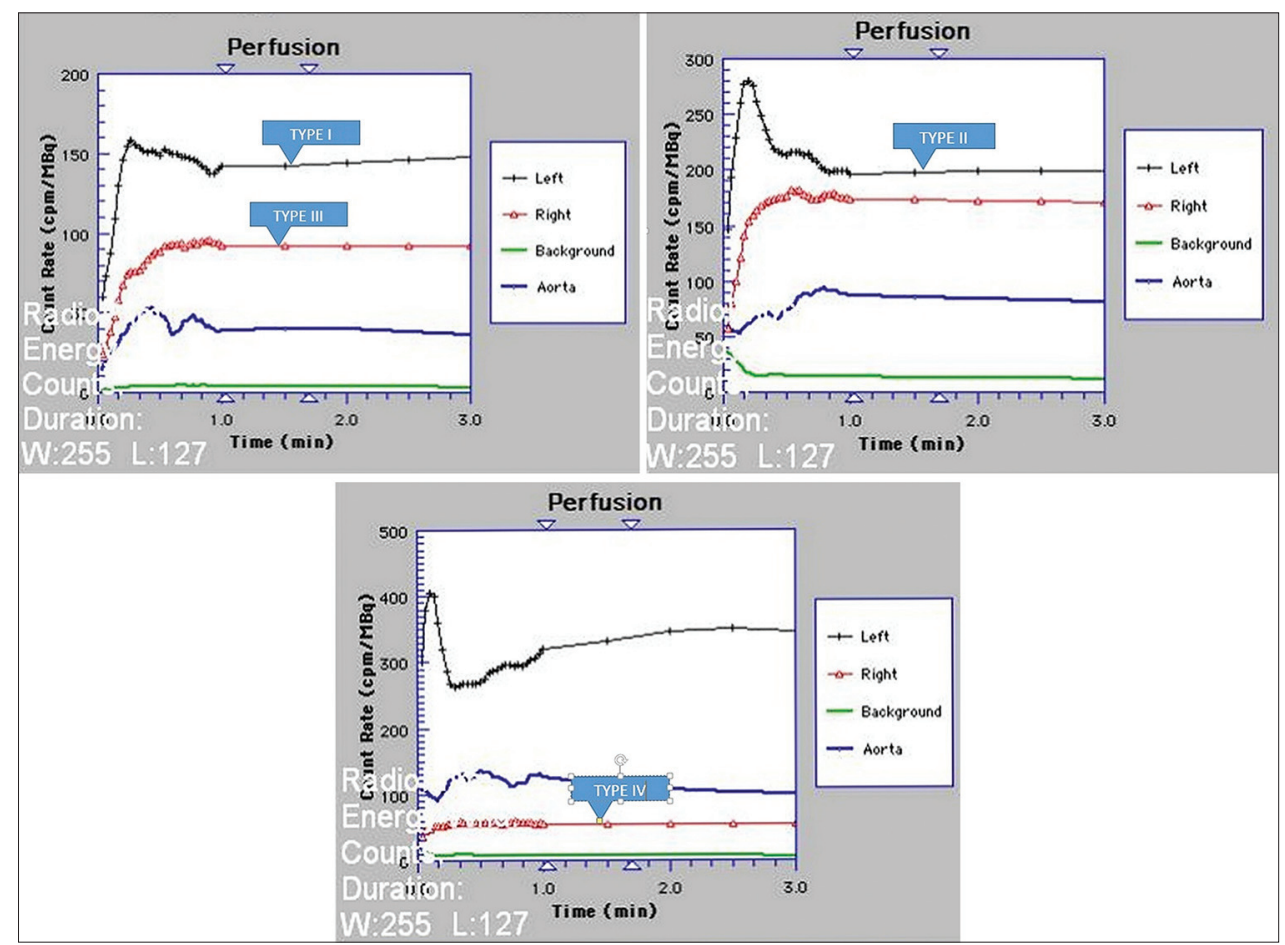

Figure 2: Actual curves obtained after processing the acquired ranal scans

TYPE II: AP > RAP, SAP RAP

Type III perfusion phase curve has a small blunted rise followed by plateau or serrations.

When aortic pressure is less than or equal to renal artery pressure and renal artery pressure is equal to segmental artery pressure than we will get a typical TYPE III curve', as shown in the Figures 2 and 4.

TYPE III: AP $\leq$ RAP $=$ SAP

Type IV perfusion phase curves are flat or have undulations.

When all the three pressure are equal than we will get typical TYPE IV curve ${ }^{9}$, as shown in the Figures 2 and 5.

TYPE IV: AP $=$ RAP $=$ SAP

The Kirchner's K/A ratio and Hilson perfusion index were calculated for different types of curves. These perfusion curves were compared with the uptake phase of the renographic curves. For 36 patients, perfusion curve for each kidney was compared with the conventional renographic curves.

Kirchner's K/A ratio and Hilson index of different kidneys were compared with the perfusion curve of the same kidney, 4 different types of curves had respectively different Kirchner's K/A ratio and Hilson index values as shown in Table 1. The different perfusion curves were verified by having the Kirchner's K/A ratio and Hilson index in same range.

\section{RESULTS}

The perfusion curves were scrutinised and categorised in 4 types. Type I, Type II, Type III, Type IV. Kirchner's $\mathrm{K} / \mathrm{A}$ ratio and Hilson perfusion index shows a good correlation with the uptake phase of conventional renal curve. Table 2 shows the total number of different types of curves observed in total population (2X32=72kidneys).

\begin{tabular}{|c|c|c|}
\hline Curve type & Kirchner's K/A ratio & Hilson index \\
\hline Type I & $3.15 \pm 0.28$ & $32.01 \pm 2.83$ \\
\hline Type II & $2.49 \pm 0.21$ & $40.43 \pm 3.26$ \\
\hline Type III & $1.98 \pm 0.37$ & $52.19 \pm 9.77$ \\
\hline Type IV & $0.86 \pm 0.45$ & $122.08 \pm 56.86$ \\
\hline
\end{tabular}

\begin{tabular}{lcc}
$\begin{array}{l}\text { Table 2: Total number of different types of } \\
\text { curves observed in total population }\end{array}$ \\
\hline Curve type & Population & Percentage \\
\hline Type I & $34 / 72$ & 47.22 \\
Type II & $16 / 72$ & 22.22 \\
Type III & $12 / 72$ & 16.67 \\
Type IV & $10 / 72$ & 13.89 \\
\hline
\end{tabular}




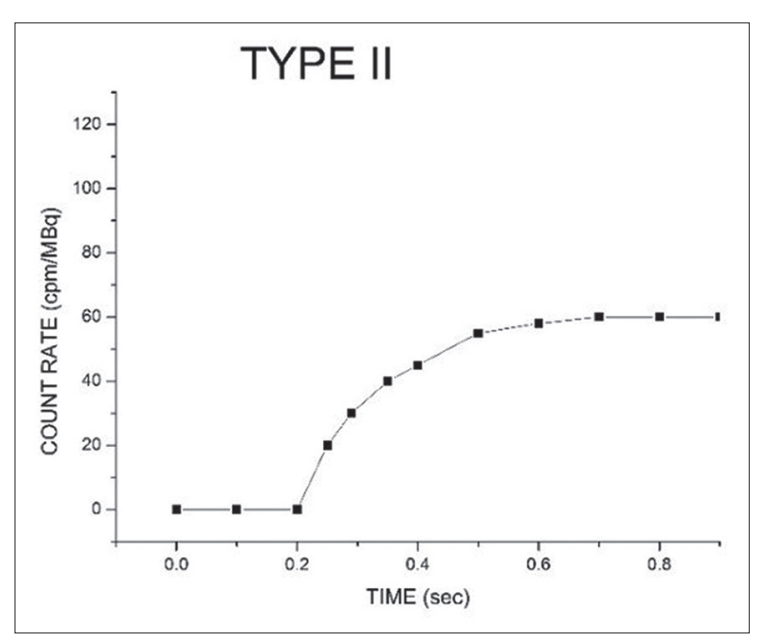

Figure 3: Ideal Type II perfusion phase curve

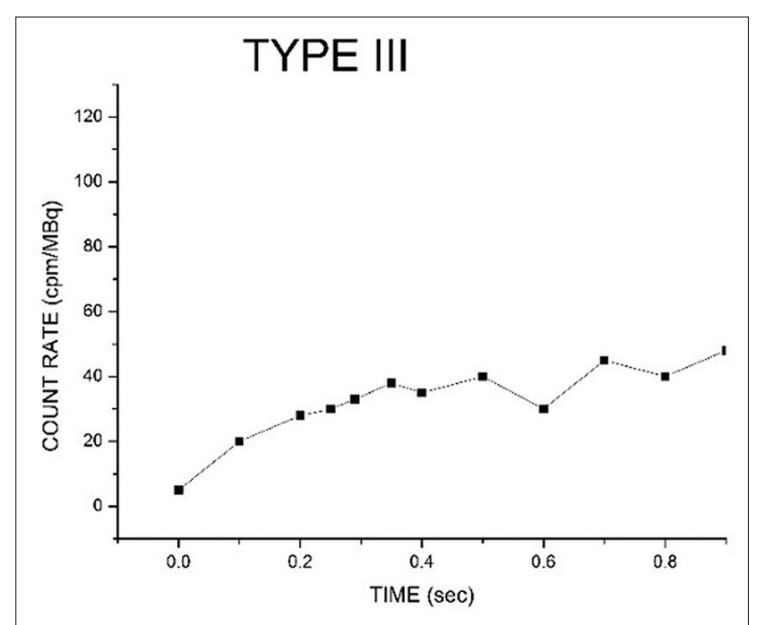

Figure 4: Ideal Type III perfusion phase curve

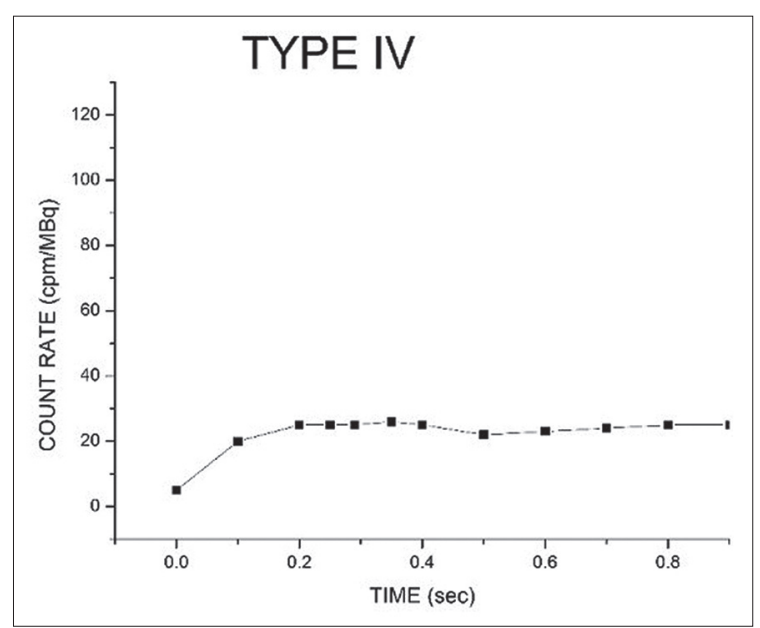

Figure 5: Ideal Type IV perfusion phase curve

The Kirchner's K/A ratio and Hilson index values, validate the characterisation of the proposed curve types.

\section{DISCUSSION}

Till now perfusion curves had importance only in the renal transplant evaluation to check the perfusion in the transplanted kidney but first time in this study we have looked into the perfusion phase of the renal curve for non-transplant kidneys. By seeing the perfusion curve, the blood flow into the renal arteries and segmental arteries can be inferred.

As In the normal condition the aortic pressure will be more than renal artery pressure which will be more than segmental artery pressure. In this case we will get the typical TYPE I curve, but When the aortic pressure is greater than renal artery pressure and the segmental artery pressure is greater than or equal to renal artery pressure then we will get a typical TYPE II curve, When aortic pressure is less than or equal to renal artery pressure and renal artery pressure is equal to segmental artery pressure then we will get a typical TYPE III curve and in the case when all the three pressure are equal then we will get typical TYPE IV curve. This defines the glomerular changes leading to vascular changes.

\section{CONCLUSIONS}

This study highlights the utility of renal perfusion phase curve analysis not only in the transplant kidney but in normal kidneys as well. This can also serve as a better prognostic tool in the chronic kidney disease patient evaluation.

\section{ACKNOWLEDGEMENT}

Authors express their gratitude to the founder chancellor of SSSIHL, Sri Sathya Sai Baba, for His constant support and Nuclear Medicine facilities at SSSIHMS.

\section{REFERENCES}

1. Kazuo । TOH. Comparison of methods for determination of glomerular filtration rate: Tc-99m-DTPA renography, predicted creatinine clearance method and plasma sample method. Annals of Nuclear Medicine 2003;17(7): 561-565.

2. Hilson AJ, Maisey MN, Brown CB, Ogg CS and Bewick MS. Dynamic renal transplant imaging with Tc-99m DTPA (Sn) supplemented by a transplant perfusion index in the management of renal transplants. J Nucl Med 1978;19:994-1000.

3. EIMaghrabya TAF, van Eck-SmitaBLF, de FijterbJW and Pauwelsa EKJ. Quantitative scintigraphic parameters for the assessment of renal transplant patients. European Journal of Radiology 1998; 28: 256-269.

4. Kirchner PT, Goldman MH, Leapman SB and Kiepfer RF. Clinical application of the kidney to aortic blood flow index (K/A ratio). Contrib Nephrol 1978; 11:120-126.

5. Hilson AJ, Maisey MN, Brown CB, Ogg CS and Bewick MS. 
Dynamic renal transplant imaging with Tc-99m DTPA (Sn) supplemented by a transplant perfusion index in the management of renal transplants. J Nucl Med 1978; 19:994-1000.

6. Kirchner PT, Goldman MH, Leapman SB and Kiepfer RF. Clinicalapplication of the kidney to aortic blood flow index (K/A ratio).Contrib Nephrol 1978; 11:120-126.

7. Kirchner PT and Rosenthall L. Renal transplant evaluation. SeminNucl Med 1982; 12:370-378.
8. Conrad GR, Wesolowski CA, Berbaum KS, Jensen KC and Kirchner PT. Renal arteriovenous transit times of technetiumradiolabeled chelates. J Nucl Med 1987; 28:1134-1143.

9. Nakamura T, Sakamaki T, Kurashina T, Sato K, Ono Z and Murata K. Effect of Renal Perfusion Pressure on Renal Interstitial Hydrostatic Pressure and Sodium Excretion, Role of Vasopressin V1 and V2 Receptors. Hypertension 1995; 25: 866-871.

\section{Authors Contribution:}

OS- Concept and design of the study, reviewed the literature, collected data statistically analysed and interpreted, prepared first draft of manuscript and critical revision of the manuscript; KVR- Concept, literature search, review of literature and critical revision of the manuscript; VSSM- Conceptualized study, literature

search, manuscript preparation, collection of data and critical revision of the manuscript. 